\title{
Network-based modular latent structure analysis
}

\author{
Tianwei $Y u^{*}$, Yun Bai ${ }^{2}$ \\ From 9th International Symposium on Bioinformatics Reseaerch and Applications (ISBRA'13) \\ Charlotte, NC, USA. 20-22 May 2013
}

\begin{abstract}
Background: High-throughput expression data, such as gene expression and metabolomics data, exhibit modular structures. Groups of features in each module follow a latent factor model, while between modules, the latent factors are quasi-independent. Recovering the latent factors can shed light on the hidden regulation patterns of the expression. The difficulty in detecting such modules and recovering the latent factors lies in the high dimensionality of the data, and the lack of knowledge in module membership.

Methods: Here we describe a method based on community detection in the co-expression network. It consists of inference-based network construction, module detection, and interacting latent factor detection from modules.

Results: In simulations, the method outperformed projection-based modular latent factor discovery when the input signals were not Gaussian. We also demonstrate the method's value in real data analysis.

Conclusions: The new method nMLSA (network-based modular latent structure analysis) is effective in detecting latent structures, and is easy to extend to non-linear cases. The method is available as R code at http://web1.sph. emory.edu/users/tyu8/nMLSA/.
\end{abstract}

\section{Background}

Modularity is a common characteristic of high-throughput biological data [1]. In a large system, the biological units, i.e. features (genes, proteins, or metabolites) are organized into quasi-autonomous modules. In expression data, each expression module can be modeled reasonably well using the latent factor approach $[2,3]$. Given the involvement of thousands of features, an unknown number of modules, and unknown module membership of the features, it is difficult to faithfully detect the modules and recover the underlying latent factors controlling the modules.

Dimension reduction methods at the global level, such as Principal Component Analysis (PCA), Independent Component Analysis (ICA) [4], sparse PCA [5,6], and Bayesian decomposition [7] are not effective in detecting localized signals. Clustering methods group co-expressed features together [8], which may help identify modules that are controlled by a single underlying signal $[9,10]$.

\footnotetext{
* Correspondence: tianwei.yu@emory.edu

'Department of Biostatistics and Bioinformatics, Rollins School of Public Health, Emory University, Atlanta, GA, USA

Full list of author information is available at the end of the article

However in real data, the features involved in the same module may not co-express when more than one latent factors control the module. We previously proposed the projection-based Modular Latent Structure Analysis (MLSA) [11], which detects modules using iteratively re-weighted singular value decomposition (SVD). So far there are no other modular decomposition methods. In this study, we seek to improve the method using a totally different approach. Our goal is to develop a method that is more intuitive, flexible, and involves less ad hoc parameter choices.

Using networks constructed from expression data can provide a flexible framework for module detection [12-14]. Here we present a method to identify modules and the underlying latent signals in three steps: (1) constructing a co-expression network based on statistical inference and local false discovery rate (lfdr); (2) detecting communities in the network; and (3) recovering interacting latent factors from the modules.

The goal of the algorithm is to achieve modular matrix decomposition. We attempt to solve the problem by assembling tools from some well-established fields. The first is the reverse engineering of genome-scale 
networks. There are a number of methods available in this area, which were designed with different objectives, including Gaussian Graphical Models where the absence of an edge signifies conditional independence $[15,16]$, co-expression network where edges signify marginal dependence [13], information theory-based networks [17], and Bayesian networks [18]. In this study, we designed our own method to estimate an inferencebased co-expression network using the local false discovery rate (lfdr) concept [19-21]. The use of local fdr makes the procedure adaptive to shifts of baseline correlation levels and avoids constructing overly dense networks when there are pervasive low-level correlations between genes. Once the network is constructed, we borrow a method from the mature field of community detection in large networks [22-25]. This is followed by latent factor extraction and rotation using factor analysis methods [26]. Added together, the assembled tools make a very good heuristic solution to the modular decomposition problem.

We demonstrate the superiority of the new method against existing modular and global decomposition methods using simulations, and apply the method to a real dataset to show it detects biologically meaningful modules that are controlled by multiple latent factors.

\section{Methods}

\section{The objective}

Given a data matrix $\boldsymbol{G}_{p \times n}$ with $p$ features measured in $n$ conditions, we seek to assign subgroups of the features into modules, such that within each module, the expression levels of the features can be modeled by a linear factor model

$$
G_{q \times n}^{(\text {module })}=L_{q \times r} F_{r \times n}+E_{q \times n}
$$

where $q$ is the size of the module, $r$ is the number of latent factors controlling the module, $L$ is the regulation strength (loading) matrix, and $\boldsymbol{E}$ is the residual matrix. Our interest is estimating (1) the number of modules, (2) the module membership of the features, (3) the activities of the latent factors controlling each module ( $\boldsymbol{F}$ matrix), and (4) the regulation strength of each factor on each feature ( $L$ matrix).

\section{The estimation procedure}

Figure 1 illustrates the procedure using a toy dataset with two modules. Generally, three steps are involved.

Step 1. Constructing co-expression network based on local fdr. We use the concept of local false discovery rate (lfdr) to establish links between features [19]. First,




we compute the correlation coefficients $r_{i j}$ between all pairs of features. Secondly, we transform the correlation coefficients by

$$
t_{i j}=r_{i j} \sqrt{(n-2) /\left(1-r_{i j}^{2}\right)},
$$

so that the distribution of the resulting statistic is close to normal under the null hypothesis that the pair of features are independent [27]. Thirdly, we compute the local false discovery rate using Efron's procedure [19]. The local fdr is a statistical statement of how likely two features are independent given we observe the statistics from all pairs of features. Fourth, if the local fdr value for a pair of features is smaller than a threshold, e.g. 0.2, an edge is established between the two features.

Step 2. Module detection in the co-expression network. We first use a well-established method that detects dense sub-graphs from a sparse graph by short random walks [25]. To fine-tune the results, we conduct an additional community-merging step. For a pair of communities $C_{i}$ that contains $m_{i}$ features and $k_{i}$ within-community connections, and $C_{j}$ that contains $m_{j}$ features and $k_{j}$ within-community connections, we divide the number of between-community connections $k_{i j}$ by the expected number of connections if the communities were indeed one

$$
\delta_{i j}=\frac{k_{i j}}{m_{i} m_{j} \times\left(k_{i}+k_{j}\right)}\left(\left(\begin{array}{c}
m_{i} \\
2
\end{array}\right)+\left(\begin{array}{c}
m_{j} \\
2
\end{array}\right)\right) .
$$

We then pool all the $\delta_{i j}$ values computed from all pairs of communities and examine the distribution. Any outlier $\delta_{i j}$, defined by a value higher than the median plus four times the difference between the $75^{\text {th }}$ percentile and the median, signifies a community pair that should be merged into a single community.

Step 3. Detecting latent factors from each module. For each module, we first conduct an eigenvalue decomposition of the covariance matrix, and select all eigenvectors that account for at least $5 \%$ of the data variance. We then find the projection length of each feature onto each eigenvector $\left\{l_{i}^{(j)}\right\}_{i=1, \ldots m_{i}, j=1, \ldots, n_{j}}$, where $i$ denotes the feature and $j$ denotes the eigenvector. The value $m_{j}$ is the number of features in the module, and $n_{j}$ is the number of eigenvectors under consideration.

Two eigenvectors are considered "interactive" if the correlation of the projection length of the features onto these two vectors is statistically significant. We initiate a selected vector set with only the first eigenvector. Then from the second eigenvector on, if the eigenvector is interactive with any vector in the selected set, it is added to the selected set. Otherwise we stop the iteration and return the selected vector set as the latent variables of the module. If more than one eigenvector is selected, we rotate them using oblique rotation [26].

Step 4. The overall factor model. After finding a collection of $\boldsymbol{F}$ matrices, we can combine them into an overall factor model with a sparse loading matrix to interpret the gene expression. Let $K$ be the total number of latent factors found, $\boldsymbol{B}$ be the combined factor activity matrix of all the factor scores, $\boldsymbol{L}$ be the loading matrix, and $\boldsymbol{E}$ be the unexplained expression, we have a factor model,

$$
G_{p \times n}=L_{p \times K} B_{K \times n}+E_{p \times n}
$$

The values in $\boldsymbol{L}$ can be filled in two ways. The first is by performing linear regression of each gene against only the factors of the modules the gene is assigned to. Alternatively, we can perform regularized regression of each gene against all the factors using lasso [28] with BIC (Bayesian information criterion) model selection.

\section{Simulation study}

We refer to our method as "Network-based Modular Latent Factor Analysis (nMLSA)". We compared the method with MLSA [11], PCA, ICA [29], factor analysis with oblique rotation [26], gene shaving [9], and sparse principal component analysis (SPCA) [5]. In each simulation, we generated a gene expression dataset with 10 modules. Every module consisted of 100 simulated genes. The number of latent factors controlling the module was randomly selected between 1 and 3. An additional 1000 pure noise genes were generated from the standard Gaussian distribution. We vary the following parameters in the simulations:

(1) The latent factor scores were either independent Gaussian, or randomly chosen from a mixture of four types: Gaussian, sine wave, square wave, and sawtooth wave (Additional file 1 Figure S1). The setting stayed the same for every module in each simulated dataset.

(2) Different levels of within-module loading sparsity, i.e. proportion of zero loadings, were tested. The sparsity of the loading matrix was achieved by drawing samples from the binomial distribution. After the nonzero positions in the loading matrix was determined, for every simulated gene, if there were $m$ controlling factors, we divided $[0,1]$ into $m$ regions by drawing ( $m$ 1) samples from the uniform distribution between 0 and 1 . We then used the sizes of the regions as the loadings for the gene. Half of the loadings were then multiplied by -1 to generate negative loadings. The sparsity levels tested were $0 \%, 30 \%$ and $60 \%$. The setting stayed the same for every module in each simulated dataset.

(3) After multiplying the loading matrix and the factor score matrix to generate the simulated expression 
matrix, Gaussian random noise was added to achieve different signal to noise ratios (values used: 1,2 ). The setting stayed the same for every module in each simulated dataset.

The number of samples was set at 100. All possible combinations of the parameters were tested, each repeated 100 times.

To judge the performance of the methods, we used the information of the true hidden factors to group the identified factors. Let $K$ be the combined hidden factor count from all modules in the simulated dataset. We first performed linear regression of every identified factor against each hidden factor group (those controlled the same module), and recorded the multiple $R^{2}$. The identified factor was assigned to the hidden factor group with which it had the largest $\mathrm{R}^{2}$ value. The $K$ identified factors with the largest $R^{2}$ values were retained for the next step. Second, we performed linear regression of every true hidden factor against the identified factors assigned to its group, and recorded the multiple $R^{2}$ as the level of recovery of the true hidden factor. The ideal method should yield multiple $\mathrm{R}^{2}$ values close to one. After repeating the simulation from every parameter setting 100 times, we compared the methods by the distribution of the multiple $R^{2}$ values.

\section{Results}

Simulation results

The simulation results are summarized in Figure 2. Each sub-plot represents a parameter setting. The relative frequencies (10 equal-sized bins between 0 and 1 , equivalent to the histogram) of the $R^{2}$ values are plotted in Figure 1. Different colors represent different methods. The curves are effectively histograms of the multiple $R^{2}$ values. The curve of a better method should show higher frequency in larger $\mathrm{R}^{2}$ values. In all the scenarios, clearly nMLSA (red) and MLSA (blue) outperformed the other methods.

When the true signals were Gaussian (Figure 2; two right columns), nMLSA and MLSA yielded similar results. Both methods recovered the hidden factors almost perfectly in all sparsity (rows) and noise (columns) settings. When the true signals were randomly drawn from four different types (Figure 2; two left columns), nMLSA outperformed MLSA. Both methods tend to either fully recover or totally miss a hidden factor, as indicated by spikes at $R^{2}=1$ and $R^{2}=0$. However when the within-module sparsity was moderate to low (30\% and $0 \%)$, nMLSA showed a roughly 3 -fold reduction in the chance to miss hidden factors, and accordingly a much higher chance to faithfully recover the hidden factors.

\section{Real data analysis}

The Spellman cell cycle data consists of four time-series, each covering roughly two cell cycles [30]. The array data consists of 73 conditions and 6178 genes. Because of phase differences, the cell cycle-related genes cannot be easily summarized by clusters although many of them exhibit periodic patterns [31]. We applied nMLSA to the cell cycle data as a whole, in order to discover common patterns across the four time series. Our method identified 7 modules containing 10 latent factors in total. The two largest modules each contained two latent factors (Figure 3).

While MLSA also detected the second module, it failed to detect module 1 found by nMLSA (Figure 3, left panel). Functional analyses using Gene Ontology [32] indicate the module is highly biologically meaningful. Based on hypergeometric tests using the GOStats package [33], genes associated with the first factor of the module strongly over-represent biological processes related to RNA processing and the ribosome, which is central to protein biosynthesis (Table 1). Genes associated with the second factor over-represent biological processes related to protein degradation, transport and localization (Table 2). Protein transport and localization processes are naturally coordinated with protein biosynthesis. Evidences also point to the co-regulation of protein biosynthesis and protein degradation, under normal circumstances and experimental interference [34-36].

The second module is even more intuitive biologically. The factor scores showed that the second module was governed by two periodic latent factors with similar periodicity but different phases (Figure 3, right). Genes of this module showed clear periodic behavior with different phase shifts (Figure 4), which is consistent with the biological knowledge that cell-cycle genes are activated at different phases of the cell cycle [30]. We analyzed the functionalities of the genes associated with each factor using gene ontology (GO). It was clear that cell cycle-related biological processes dominated the list of top processes overrepresented by genes associated with either latent factors (Tables $3 \& 4$ ). Other methods used in the simulations, except MLSA, could not group cell cycle genes with different phase shift into a single module.

\section{Discussions}

In this study, we developed the network-based modular latent structure analysis (nMLSA). It is aimed at detecting expression modules and latent factors controlling the modules, the same goal as the original MLSA [11]. Compared to MLSA, the new method is based on a totally different setup, and is substantially advantageous. Firstly, 

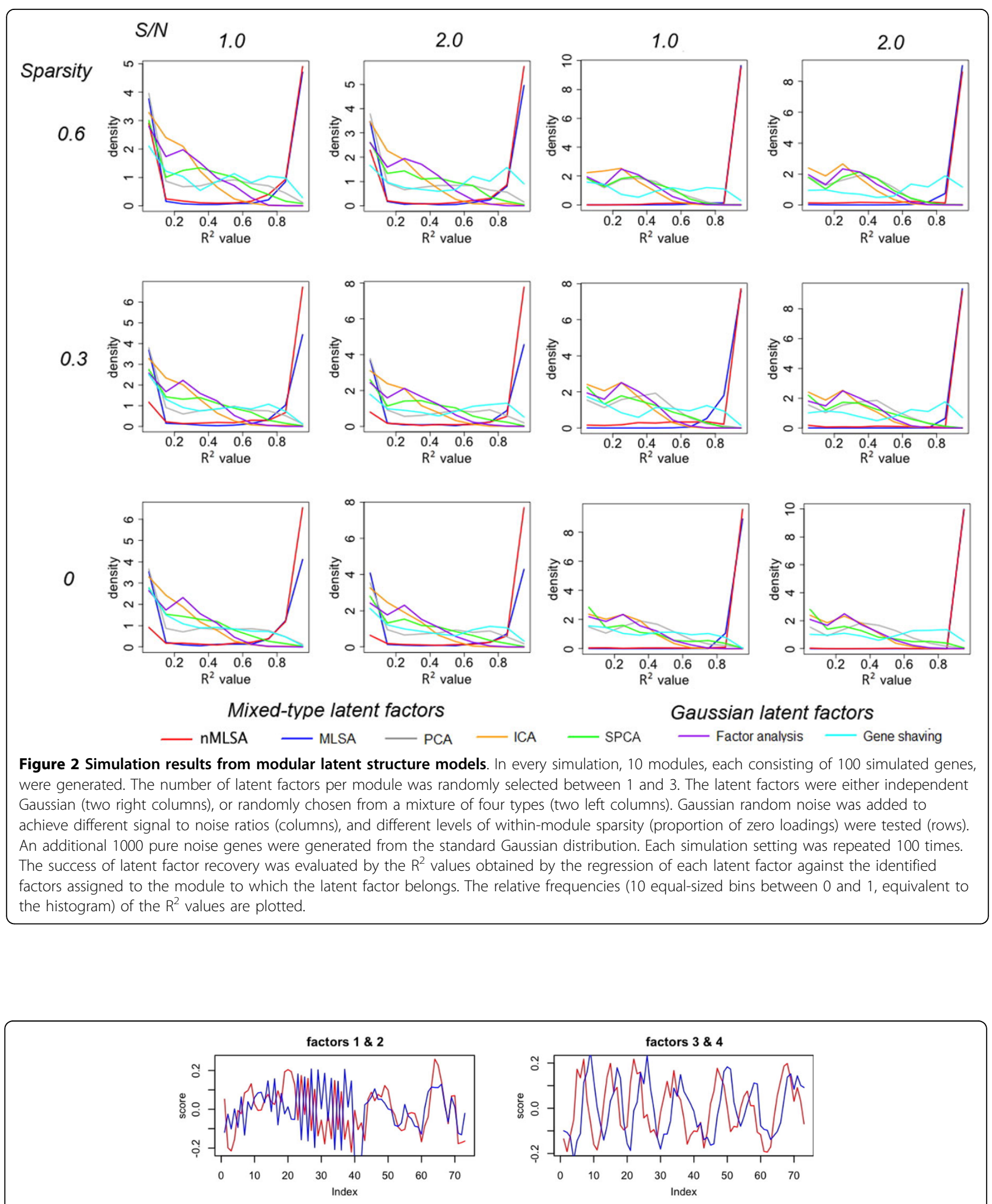

Figure 3 The top four factors from the Spellman dataset form two modules. On the $x$-axis the four time series are displayed in sequential order. 
Table 1 Top 25 GO terms overrepresented by genes associated with factor 1 .

\begin{tabular}{|c|c|c|}
\hline GOBPID & Pvalue & Term \\
\hline GO:0042254 & $3.27 \mathrm{E}-75$ & ribosome biogenesis \\
\hline GO:0022613 & $2.04 \mathrm{E}-68$ & ribonucleoprotein complex biogenesis \\
\hline GO:0034470 & $6.20 \mathrm{E}-67$ & ncRNA processing \\
\hline GO:0034660 & $1.31 \mathrm{E}-65$ & ncRNA metabolic process \\
\hline GO:0006364 & $1.55 \mathrm{E}-60$ & rRNA processing \\
\hline GO:0016072 & $1.47 \mathrm{E}-59$ & rRNA metabolic process \\
\hline GO:0006396 & $3.00 \mathrm{E}-51$ & RNA processing \\
\hline GO:0071843 & $1.48 \mathrm{E}-50$ & cellular component biogenesis at cellular level \\
\hline GO:0042273 & $5.74 \mathrm{E}-33$ & ribosomal large subunit biogenesis \\
\hline GO:0000460 & $4.04 \mathrm{E}-30$ & maturation of $5.8 \mathrm{~S}$ rRNA \\
\hline GO:0000466 & $2.05 \mathrm{E}-29$ & maturation of $5.8 \mathrm{~S}$ rRNA from tricistronic rRNA transcript (SSU-rRNA, $5.8 \mathrm{~S}$ rRNA, LSU-rRNA) \\
\hline GO:0016070 & $1.33 \mathrm{E}-26$ & RNA metabolic process \\
\hline GO:0042274 & 4.46E-26 & ribosomal small subunit biogenesis \\
\hline GO:0044085 & $3.25 \mathrm{E}-25$ & cellular component biogenesis \\
\hline GO:0030490 & $1.57 \mathrm{E}-21$ & maturation of SSU-rRNA \\
\hline GO:0000462 & $3.11 \mathrm{E}-21$ & maturation of SSU-rRNA from tricistronic rRNA transcript (SSU-rRNA, $5.8 \mathrm{~S}$ rRNA, LSU-rRNA) \\
\hline GO:0000469 & $3.59 \mathrm{E}-21$ & cleavage involved in rRNA processing \\
\hline GO:0010467 & $7.58 \mathrm{E}-21$ & gene expression \\
\hline GO:0090304 & $2.29 \mathrm{E}-16$ & nucleic acid metabolic process \\
\hline GO:0009451 & $2.58 \mathrm{E}-16$ & RNA modification \\
\hline GO:0000478 & $8.00 \mathrm{E}-16$ & endonucleolytic cleavage involved in rRNA processing \\
\hline GO:0000479 & $8.00 \mathrm{E}-16$ & endonucleolytic cleavage of tricistronic rRNA transcript (SSU-rRNA, 5.85 rRNA, LSU-rRNA) \\
\hline GO:0006139 & 1.09E-15 & nucleobase-containing compound metabolic process \\
\hline GO:0000447 & 1.37E-15 & $\begin{array}{l}\text { endonucleolytic cleavage in ITS1 to separate SSU-rRNA from } 5.8 S \text { rRNA and LSU-rRNA from tricistronic } \\
\text { rRNA transcript (SSU-rRNA, 5.8S rRNA, LSU-rRNA) }\end{array}$ \\
\hline GO:0000472 & $1.42 \mathrm{E}-14$ & endonucleolytic cleavage to generate mature $5^{\prime}$-end of SSU-rRNA from (SSU-rRNA, 5.8 S rRNA, LSU-rRNA) \\
\hline
\end{tabular}

Table 2 Top 25 GO terms overrepresented by genes associated with factor 2.

\begin{tabular}{lll}
\hline GOBPID & Pvalue & Term \\
\hline GO:0006511 & $1.99 \mathrm{E}-09$ & ubiquitin-dependent protein catabolic process \\
GO:0019941 & $2.44 \mathrm{E}-09$ & modification-dependent protein catabolic process \\
GO:0044257 & $2.65 \mathrm{E}-09$ & cellular protein catabolic process \\
GO:0051603 & $3.79 \mathrm{E}-09$ & proteolysis involved in cellular protein catabolic process \\
GO:0043632 & $4.21 \mathrm{E}-09$ & modification-dependent macromolecule catabolic process \\
GO:0030163 & $7.26 \mathrm{E}-09$ & protein catabolic process \\
GO:0010499 & $2.24 \mathrm{E}-08$ & proteasomal ubiquitin-independent protein catabolic process \\
$\mathrm{GO}: 0044265$ & $9.94 \mathrm{E}-08$ & cellular macromolecule catabolic process \\
$\mathrm{GO}: 0007005$ & $1.38 \mathrm{E}-07$ & mitochondrion organization \\
$\mathrm{GO}: 0043623$ & $2.59 \mathrm{E}-07$ & cellular protein complex assembly \\
$\mathrm{GO}: 0043248$ & $3.56 \mathrm{E}-07$ & proteasome assembly \\
$\mathrm{GO}: 0009057$ & $5.19 \mathrm{E}-07$ & macromolecule catabolic process \\
$\mathrm{GO}: 0006508$ & $5.25 \mathrm{E}-07$ & proteolysis \\
$\mathrm{GO}: 0071842$ & $3.02 \mathrm{E}-06$ & cellular component organization at cellular level \\
$\mathrm{GO}: 0015031$ & $4.89 \mathrm{E}-06$ & protein transport \\
$\mathrm{GO}: 0008104$ & $6.28 \mathrm{E}-06$ & protein localization \\
$\mathrm{GO}: 0043161$ & $6.81 \mathrm{E}-06$ & proteasomal ubiquitin-dependent protein catabolic process \\
$\mathrm{GO}: 0045184$ & $7.86 \mathrm{E}-06$ & establishment of protein localization \\
$\mathrm{GO}: 0010498$ & $1.01 \mathrm{E}-05$ & proteasomal protein catabolic process \\
$\mathrm{GO}: 0006461$ & $1.35 \mathrm{E}-05$ & protein complex assembly \\
$\mathrm{GO}: 0034613$ & $1.41 \mathrm{E}-05$ & cellular protein localization
\end{tabular}


Table 2 Top 25 GO terms overrepresented by genes associated with factor 2. (Continued)

\begin{tabular}{lll}
\hline GO:0070271 & $2.51 \mathrm{E}-05$ & protein complex biogenesis \\
$\mathrm{GO}: 0070727$ & $2.56 \mathrm{E}-05$ & cellular macromolecule localization \\
$\mathrm{GO}: 0034621$ & $3.00 \mathrm{E}-05$ & cellular macromolecular complex subunit organization \\
$\mathrm{GO}: 0009987$ & $3.93 \mathrm{E}-05$ & cellular process \\
\hline
\end{tabular}

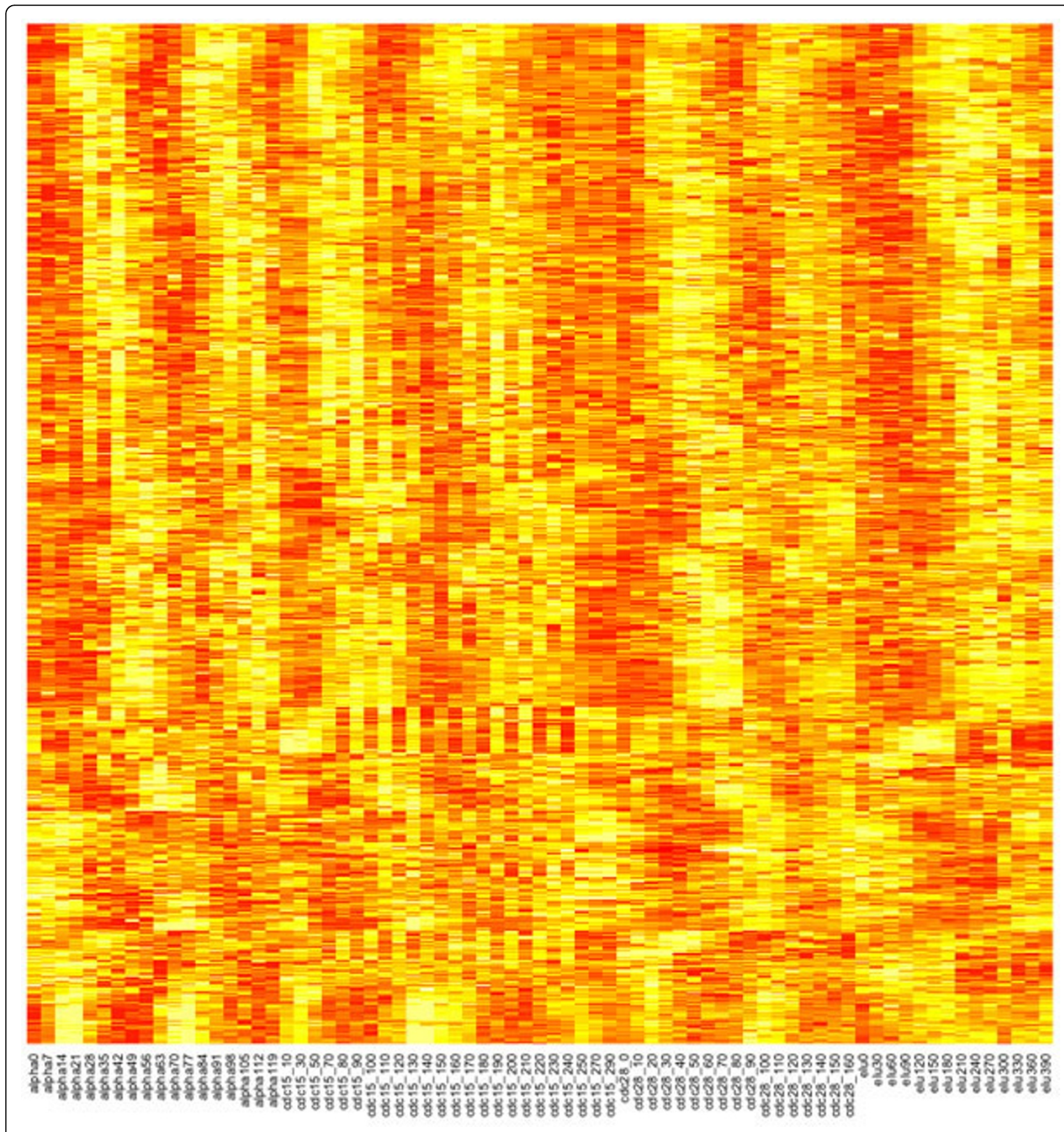

Figure 4 Expression levels of genes involved in module 2. Genes are hierarchically clustered. The four time series are displayed in sequential order. 
Table 3 Top 25 GO terms overrepresented by genes associated with factor 3 .

\begin{tabular}{|c|c|c|}
\hline GOBPID & Pvalue & Term \\
\hline GO:0000278 & $4.02 \mathrm{E}-19$ & mitotic cell cycle \\
\hline GO:0022402 & 4.08E-17 & cell cycle process \\
\hline GO:0007049 & $6.05 \mathrm{E}-17$ & cell cycle \\
\hline GO:0022403 & $5.72 \mathrm{E}-15$ & cell cycle phase \\
\hline GO:0007017 & 8.75E-15 & microtubule-based process \\
\hline GO:0048285 & $2.31 \mathrm{E}-13$ & organelle fission \\
\hline GO:0000280 & $5.08 \mathrm{E}-13$ & nuclear division \\
\hline GO:0051301 & 8.45E-13 & cell division \\
\hline GO:0000226 & $8.50 \mathrm{E}-13$ & microtubule cytoskeleton organization \\
\hline GO:0000087 & $9.22 \mathrm{E}-13$ & M phase of mitotic cell cycle \\
\hline GO:0007067 & 1.39E-12 & mitosis \\
\hline GO:0000279 & 2.71E-09 & $M$ phase \\
\hline GO:0007010 & $1.75 \mathrm{E}-08$ & cytoskeleton organization \\
\hline GO:0007059 & 2.33E-08 & chromosome segregation \\
\hline GO:0030472 & $3.11 \mathrm{E}-08$ & mitotic spindle organization in nucleus \\
\hline GO:0032886 & 3.46E-08 & regulation of microtubule-based process \\
\hline GO:0070507 & 3.46E-08 & regulation of microtubule cytoskeleton organization \\
\hline GO:0016043 & 5.99E-08 & cellular component organization \\
\hline GO:0007051 & 7.48E-08 & spindle organization \\
\hline GO:0051329 & 7.69E-08 & interphase of mitotic cell cycle \\
\hline GO:0007052 & 1.09E-07 & mitotic spindle organization \\
\hline GO:0051325 & $1.12 \mathrm{E}-07$ & interphase \\
\hline GO:0006928 & 1.15E-07 & cellular component movement \\
\hline GO:0007018 & 2.33E-07 & microtubule-based movement \\
\hline GO:0010564 & $6.27 \mathrm{E}-07$ & regulation of cell cycle process \\
\hline
\end{tabular}

Table 4 Top 25 GO terms overrepresented by genes associated with factor 4 .

\begin{tabular}{lll}
\hline GOBPID & Pvalue & Term \\
\hline GO:0007049 & $5.54 \mathrm{E}-08$ & cell cycle \\
GO:0051301 & $8.21 \mathrm{E}-08$ & cell division \\
GO:0000278 0000087 & $7.99 \mathrm{E}-07$ & mitotic cell cycle \\
GO:0022402 & $1.12 \mathrm{E}-06$ & M phase of mitotic cell cycle \\
GO:0022403 & $1.57 \mathrm{E}-06$ & cell cycle process \\
GO:0048285 & $6.92 \mathrm{E}-06$ & cell cycle phase \\
GO:0000280 & $2.16 \mathrm{E}-05$ & organelle fission \\
GO:0010458 & $3.03 \mathrm{E}-05$ & nuclear division \\
GO:0000910 & $3.55 \mathrm{E}-05$ & exit from mitosis \\
GO:0000279 & $3.70 \mathrm{E}-05$ & Cytokinesis \\
GO:0007067 & $4.43 \mathrm{E}-05$ & M phase \\
GO:0033205 & $7.91 \mathrm{E}-05$ & Mitosis \\
GO:0032506 & 0.000523865 & cell cycle cytokinesis \\
GO:0010970 & 0.000572574 & cytokinetic process \\
GO:0030473 & 0.001012686 & microtubule-based transport \\
GO:0030705 & 0.001012686 & nuclear migration along microtubule \\
GO:0072384 & 0.001012686 & cytoskeleton-dependent intracellular transport \\
GO:0000114 & 0.001012686 & organelle transport along microtubule \\
GO:0046459 & 0.00177365 & regulation of transcription involved in G1 phase of mitotic cell cycle \\
GO:0007018 & 0.00185316 & short-chain fatty acid metabolic process \\
GO:0016575 & 0.002062712 & microtubule-based movement \\
& 0.002062712 & histone deacetylation
\end{tabular}


Table 4 Top 25 GO terms overrepresented by genes associated with factor 4. (Continued)

\begin{tabular}{lll}
\hline GO:0032392 & 0.002399826 & DNA geometric change \\
GO:0010696 & 0.002438214 & positive regulation of spindle pole body separation \\
GO:0007097 & 0.002544351 & nuclear migration \\
\hline
\end{tabular}

the number of tuning parameters and heuristic choices is substantially less compared to MLSA. Secondly, the method is much more intuitive to understand. Thirdly, it is more flexible. As an example, one can easily limit the gene relations to positive correlations and ignore negative correlations using nMLSA, while MLSA has to take both positive and negative correlations. Fourth, nMLSA can be adapted for nonlinearly associated modules if a nonlinear association measure is used in the co-expression network building, while MLSA is limited to linear relations. In the nonlinear case, it is difficult to define latent factors. The challenge is subject to our future studies.

Instead of using hard cutoffs, nMLSA utilizes the concept of local false discovery rate (lfdr). As different datasets exhibit different levels of baseline correlation [37], using hard cutoffs on correlations may result in unsatisfactory results. Using local false discovery rate procedures that are flexible in the null distribution estimation, nMLSA is naturally adaptive to the characteristics of the data. Given the nMLSA procedure relies on existing network community detection algorithms, it is admitted that the performance of the method relies on the choice of the community detection algorithm. The research field of community detection is mature and a number of good methods are available. Thus it is not difficult to tune the method to achieve good performance.

\section{Conclusions}

In summary, the new network-based method nMLSA is more effective than existing methods in recovering biologically meaningful latent variables and latent variable groups. The method can potentially be extended to detect nonlinearly associated modules if a nonlinear association measure is used to build the network.

\section{Additional material}

Additional file 1: Figure S1. The four types of input signal from which the data were simulated.

Competing interests

None.

\section{Authors' contributions}

TY developed the computational method, conducted simulations. TY and YB conducted real data analyses, interpreted the results, and drafted the manuscript.

\section{Acknowledgements}

This work was partially supported by NIH grants P20HL113451, P30AI50409 and U19AI090023. The funding source to publish the publication cost is $\mathrm{NIH}$ grant U19A1090023.

This article has been published as part of BMC Bioinformatics Volume 15 Supplement 13, 2014: Selected articles from the 9th International Symposium on Bioinformatics Research and Applications (ISBRA'13): Bioinformatics. The full contents of the supplement are available online at http://www.biomedcentral.com/bmcbioinformatics/supplements/15/S13.

\section{Authors' details}

${ }^{1}$ Department of Biostatistics and Bioinformatics, Rollins School of Public Health, Emory University, Atlanta, GA, USA. ${ }^{2}$ Department of Pharmaceutical Sciences, School of Pharmacy, Philadelphia College of Osteopathic Medicine, Suwanee, GA, USA.

Published: 13 November 2014

\section{References}

1. Wagner GP, Pavlicev M, Cheverud JM: The road to modularity. Nat Rev Genet 2007, 8:(12):921-931.

2. Yu T, Li KC: Inference of transcriptional regulatory network by two-stage constrained space factor analysis. Bioinformatics 2005, 21(21):4033-4038.

3. Liao JC, Boscolo R, Yang YL, Tran LM, Sabatti C, Roychowdhury VP: Network component analysis: reconstruction of regulatory signals in biological systems. Proc Natl Acad Sci USA 2003, 100(26):15522-15527.

4. Lee TW: Independent component analysis : theory and applications. Boston: Kluwer Academic Publishers; 1998.

5. Zou H, Hastie T, Tibshirani R: Sparse principal component analysis. Journal of Computational and Graphical Statistics 2006, 15(2):265-286.

6. Carvalho CM, Chang J, Lucas JE, Nevins JR, Wang Q, West M: HighDimensional Sparse Factor Modeling: Applications in Gene Expression Genomics. Journal of the American Statistical Association 2008, 103(484):1438-1456

7. Moloshok TD, Klevecz RR, Grant JD, Manion FJ, Speier WFt, Ochs MF: Application of Bayesian decomposition for analysing microarray data. Bioinformatics 2002, 18(4):566-575.

8. Gan G, Ma C, Wu J: Data clustering : theory, algorithms, and applications. Philadelphia, Pa. Alexandria, Va.: SIAM. American Statistical Association; 2007.

9. Hastie T, Tibshirani R, Eisen MB, Alizadeh A, Levy R, Staudt L, Chan WC, Botstein D, Brown P: 'Gene shaving' as a method for identifying distinct sets of genes with similar expression patterns. Genome Biol 2000, 1(2): RESEARCH0003.

10. Yuan S, Li KC: Context-dependent clustering for dynamic cellular state modeling of microarray gene expression. Bioinformatics 2007, 23(22):3039-3047.

11. Yu T: An exploratory data analysis method to reveal modular latent structures in high-throughput data. BMC Bioinformatics 2010, 11:440.

12. Fu Q, Lemmens $K$, Sanchez-Rodreiquez A, Thijs IM, Meysman P, Sun H, Fierro AC, Engelen K, Marchal K: Directed module detection in a largescale expression compendium. Methods Mol Biol 2012, 804:131-165.

13. Langfelder $P$, Horvath S: WGCNA: an R package for weighted correlation network analysis. BMC Bioinformatics 2008, 9:559.

14. Li A, Horvath S: Network module detection: Affinity search technique with the multi-node topological overlap measure. BMC Res Notes 2009, 2:142.

15. Peng J, Wang P, Zhou N, Zhu J: Partial Correlation Estimation by Joint Sparse Regression Models. J Am Stat Assoc 2009, 104(486):735-746.

16. Schafer J, Strimmer K: An empirical Bayes approach to inferring largescale gene association networks. Bioinformatics 2005, 21(6):754-764.

17. Margolin AA, Nemenman I, Basso K, Wiggins C, Stolovitzky G, Dalla Favera $\mathrm{R}$, Califano A: ARACNE: an algorithm for the reconstruction of gene regulatory networks in a mammalian cellular context. BMC Bioinformatics 2006, 7(Suppl 1):S7. 
18. Ellis $B$, Wong W: Learning causal bayesian network structures from experimental data. Journal of the American Statistical Association 2008, 103:778-789.

19. Efron B, Tibshirani R: Empirical Bayes methods and false discovery rates for microarrays. Genet Epidemiol 2002, 23(1):70-86.

20. Strimmer K: A unified approach to false discovery rate estimation. BMC Bioinformatics 2008, 9:303.

21. Guedj M, Robin S, Celisse A, Nuel G: Kerfdr: a semi-parametric kernelbased approach to local false discovery rate estimation. $B M C$ Bioinformatics 2009, 10:84.

22. Ball B, Karrer B, Newman ME: Efficient and principled method for detecting communities in networks. Physical review E, Statistical, nonlinear, and soft matter physics 2011, 84(3 Pt 2):036103.

23. Hofman JM, Wiggins CH: Bayesian approach to network modularity. Physical review letters 2008, 100(25):258701.

24. Newman ME: Spectral methods for community detection and graph partitioning. Physical review E, Statistical, nonlinear, and soft matter physics 2013, 88(4):042822.

25. Pons $P$, Latapy M: Computing Communities in Large Networks Using Random Walks. Lecture Notes in Computer Science 2005, 3733:284-293.

26. Bernaards CA, Jennrich Rl: Gradient Projection Algorithms and Software for Arbitrary Rotation Criteria in Factor Analysis. Educational and Psychological Measurement 2005, 65:676-696.

27. Rahman NA: A course in theoretical statistics for sixth forms, technical colleges, colleges of education, universities. London;: Griffin; 1968.

28. Efron B, Hastie T, Johnstone I, Tibshirani R: Least Angle Regression. Annals of Statistics 2003, 32(2):407-499

29. Hastie T, Tibshirani R, Friedman JH: The elements of statistical learning : data mining, inference, and prediction. New York, NY: Springer; 22009.

30. Spellman PT, Sherlock G, Zhang MQ, lyer VR, Anders K, Eisen MB, Brown PO, Botstein D, Futcher B: Comprehensive identification of cell cycleregulated genes of the yeast Saccharomyces cerevisiae by microarray hybridization. Mol Biol Cell 1998, 9(12):3273-3297.

31. Li KC, Yan M, Yuan SS: A simple statistical model for depicting the cdc15synchronized yeast cell-cycle regulated gene expression data. Stat Sinica 2002, 12(1):141-158.

32. Ashburner M, Ball CA, Blake JA, Botstein D, Butler H, Cherry JM, Davis AP, Dolinski K, Dwight SS, Eppig JT, et al: Gene ontology: tool for the unification of biology. The Gene Ontology Consortium. Nat Genet 2000, 25(1):25-29.

33. Falcon $\mathrm{S}$, Gentleman R: Using GOstats to test gene lists for $\mathrm{GO}$ term association. Bioinformatics 2007, 23(2):257-258.

34. Dai CL, Shi J, Chen Y, Iqbal K, Liu F, Gong CX: Inhibition of protein synthesis alters protein degradation through activation of protein kinase B (AKT). J Biol Chem 2013.

35. Hemmerlin A: Post-translational events and modifications regulating plant enzymes involved in isoprenoid precursor biosynthesis. Plant Sci 2013, 203-204:41-54

36. Stein $H$, Honig A, Miller G, Erster O, Eilenberg H, Csonka LN, Szabados L, Koncz C, Zilberstein A: Elevation of free proline and proline-rich protein levels by simultaneous manipulations of proline biosynthesis and degradation in plants. Plant Sci 2011, 181(2):140-150.

37. Yu T, Zhao Y, Shen S: AAPL: Assessing Association between P-value Lists. Statistical analysis and data mining 2013, 6(2):144-155.

\section{Submit your next manuscript to BioMed Central and take full advantage of:}

- Convenient online submission

- Thorough peer review

- No space constraints or color figure charges

- Immediate publication on acceptance

- Inclusion in PubMed, CAS, Scopus and Google Scholar

- Research which is freely available for redistribution

Submit your manuscript at www.biomedcentral.com/submit 